\title{
Modos de ondas magnetohidrodinámicas influenciados por la corriente de desplazamiento
}

\author{
Magnetohydrodynamic wave modes influenced by displacement current
}

\author{
A. Ojeda-González ${ }^{* 1 @}$, J. J. González-Avilés ${ }^{2}$, V. De La Luz ${ }^{4}$, L. Nunes dos Santos ${ }^{1,2}$ \\ ${ }^{1}$ Universidade do Vale do Paraíba, Instituto de Pesquisa e Desenvolvimento, São José dos Campos, SP, Brasil. \\ ${ }^{2}$ Universidade Federal de Mato Grosso do Sul, Departamento de Matemática CPAQ, 79200-000, Aquidauana, MS, Brasil. \\ ${ }^{3}$ Cátedras CONACYT, Servicio de Clima Espacial México (SCIESMEX), Laboratorio Nacional de Clima Espacial \\ (LANCE), Instituto de Geofísica, Unidad de Michoacán, Universidad Nacional Autónoma de México, C.P. 58910, Morelia, \\ Michoacán, México. \\ ${ }^{4}$ Universidad Nacional Autónoma de México, Escuela Nacional de Estudios Superiores, Unidad Morelia, C.P. 58190, \\ Morelia, Michoacán, México.
}

Recebido em 06 de Agosto de 2020. Revisado em 05 de Março de 2021. Aceito em 31 de Março de 2021.

\begin{abstract}
En este artículo trabajamos con la ecuación de velocidad de un fluido magneto-conductor utilizando dos aproximaciones: (i) la ley de Ampère y (ii) tomando en cuenta la corrección de Maxwell. Considerando una dirección arbitraria entre el vector de onda y el campo magnético se obtiene una expresión para la velocidad de fase de la onda. Existen tres modos de onda magnetohidrodinámicas (MHD) cuando la corriente de desplazamiento $\left(J_{d}\right)$ no es considerada. Primero, se hace una comparación de los modos de onda entre sí, y después se comparan con los modos obtenidos con y sin la $J_{d}$. La comparación, permite discutir las variaciones entre estos modos MHD en los casos teóricos, cuando la velocidad de Alfvén aumenta hasta valores cercanos a la velocidad de la luz. El objetivo principal del artículo es mostrar la importancia del efecto de la $J_{d}$ en los modos puro y rápido de ondas MHD, en el caso particular en donde la velocidad de Alfvén es mayor que la velocidad adiabática de una onda acústica. Encontramos que para el modo lento, la $J_{d}$ prácticamente no influye en el valor de la velocidad de fase de la onda cuando la velocidad de Alfvén aumenta.
\end{abstract}

Palabras clave: Ondas MHD, Velocidad de Alfvén, Corriente de desplazamiento, Ecuaciones básicas de la MHD.

In this article, we work with the basic equation for the fluid velocity in the case of a magnetoconducting fluid in two ways: (i) by Ampère's law, (ii) taking into account the Maxwell correction. Considering an arbitrary direction between the wave vector and the magnetic field, an expression for the wave's phase velocity is obtained. The phase velocity study implies three magnetohydrodynamic (MHD) wave modes when we neglect the displacement current. We compared the wave modes with each other, and we also compared the modes obtained with and without the displacement current. The comparison allows us to discuss the variations between these MHD modes in hypothetical cases when the Alfvén speed increases to values close to the speed of light. The article's main objective is to show the importance of displacement current in the pure and fast MHD waves, in the particular case where the Alfvén speed is higher than the sound wave speed. We find that the displacement current has practically no influence on the value of the wave phase velocity when the Alfvén velocity increases for slow mode. Keywords: MHD waves, Alfven speed, Displacement current, Basic MHD equations.

\section{Introducción}

Un fluido no conductor puede sufrir compresiones (cambios en la presión) y rarefacciones (cambios en la densidad) cuando se propagan ondas longitudinales a través de él. Tales ondas tienen la característica de que el vector de onda $\vec{k}$ es paralelo a su velocidad de propagación [1]. Estas ondas longitudinales se pueden interpretar como acústicas con velocidad adiabática $v_{s}=\left(\gamma k_{B} T / m\right)^{1 / 2}$, siendo $\gamma$ el coeficiente de expansión adiabático, $k_{B}$ la constante de Boltzmann, $T$ la temperatura y $m$ la masa de los átomos del fluido.

\footnotetext{
*Endereço de correspondência: ojeda.gonzalez.a@gmail.com
}

En el caso de un fluido conductor bajo los efectos de un campo magnético, las partículas pueden oscilar libremente en la dirección de las líneas de campo magnético [2]. Este flujo de partículas libres hace que puedan aparecer ondas longitudinales tal y como se discutió en el párrafo anterior.

Debido a la alta conductividad (baja resistividad) del fluido magneto-conductor, se dice que las partículas se mantienen "congeladas" a las líneas del campo 3, 4, 7-10. Este "congelamiento" hace posible que surjan otro tipo de ondas, las cuales están relacionadas con la oscilación en baja frecuencia de las líneas de campo, similar a lo que ocurre en las cuerdas en vibración de una guitarra 9 , 11. 
La analogía física al considerar las líneas de campo magnético como cuerdas, implica que estas ondas deben ser transversales. Es decir, el vector de onda $\vec{k}$ es perpendicular a su velocidad de propagación. Por ejemplo, si consideramos un tubo de flujo magnético, existirá una fuerza de tensión por unidad de área de valor $B^{2} / \mu_{0}$, en las dos extremidades del tubo que estiran las líneas [12], donde $\mu_{0}$ es la permeabilidad magnética en el vacío. Además, por la presencia de los tubos vecinos, una presión isotrópica (la cual no depende de la dirección) actúa sobre el tubo de flujo haciéndolo oscilar transversalmente con una baja frecuencia. Esta oscilación transversal de las líneas se desplaza en forma de onda con una velocidad $v_{A}$, conocida como la velocidad de Alfvén (en honor al científico Hannes O. G. Alfvén, quién las descubrió en 1942 [3, 4, 13]). Estas ondas no causan compresiones ni rarefacciones en el fluido magneto-conductor [14]. La expresión matemática para calcular $v_{A}$ es:

$$
\begin{aligned}
v_{A} & =\left(\frac{\text { fuerza de tensión por unidad de área }}{\text { densidad del fluido }}\right)^{1 / 2} \\
& =\frac{B}{\sqrt{\mu_{0} \rho_{m}}},
\end{aligned}
$$

donde $\rho_{m}$ la densidad del fluido. Las ondas de Alfvén se pueden encontrar, por ejemplo, en la atmósfera solar, el viento solar, la magnetosfera de la Tierra, plasmas astrofísicos y los producidos en laboratorios [5, 15 18].

Generalmente, en un fluido magneto-conductor el campo magnético no es uniforme en todo el espacio ocupado por el fluido. La presión puede identificarse como presión cinética

$$
p=n k_{B} T
$$

donde $n$ es la densidad numérica y la presión magnética

$$
p_{B}=B^{2} / 2 \mu_{0},
$$

las cuales, en algunas regiones, pueden ser diferentes entre sí y transversales a las líneas del campo.

Estas presiones producen variaciones en la velocidad del fluido, formando ondas longitudinales magnetoacústicas, que se desplazan perpendicularmente a las líneas de campo con una velocidad igual a

$$
v_{M}^{2}=v_{s}^{2}+v_{A}^{2}
$$

En las ondas magneto-acústicas, $\vec{k}$ es paralelo a $\vec{v}_{M}$ y ambos son perpendiculares a $\vec{B}$.

El objetivo de este trabajo es mostrar los pasos algebraicos para obtener la ecuación de la velocidad de un fluido magneto-conductor de forma detallada y siguiendo un procedimiento metodológico. Esta ecuación será obtenida en un primer momento despreciando la corriente de desplazamiento en la ley de Ampère. Posteriormente será incluida la corriente de desplazamiento. Considerando una dirección arbitraria entre el vector de onda y el campo magnético en ambas ecuaciones, se obtendrá una expresión para la velocidad de fase de la onda. El estudio de la velocidad de fase implica la existencia de tres modos de ondas MHD que son ampliamente conocidos en la literatura (ver por ejemplo [19, 20, y los Capítulos 2 y 8 de [5]). Las velocidades de fase de los tres modos de ondas serán comparadas entre sí y con los obtenidos para cada una de las dos ecuaciones del fluido, con y sin corriente de desplazamiento.

La inclusión de la corriente de desplazamiento en [6] posibilitó modelar la atmósfera solar considerándola en un estado termodinámico isotérmico, para adaptar un problema de frontera al estudio de la propagación de ondas de Alfvén. La corriente de desplazamiento también debe ser considerada cuando se estudian las ondas de Alfvén en plasmas astrofísicos, como por ejemplo el caso de los pulsares, principalmente en la región dominada por el intenso campo magnético alrededor de la estrella de neutrones donde se origina su radiación periódica [5]. Los resultados nos permitirán discutir las variaciones entre estos modos MHD en los casos teóricos, cuando la velocidad de Alfvén aumenta hasta valores cercanos a la velocidad de la luz, causados por aumento del campo magnético o disminución de la densidad. Uno de los resultados novedosos de este trabajo es el estudio de las expresiones para la velocidad de fase de onda a partir de considerar o excluir la corriente de desplazamiento. El enfoque de este artículo es educativo, por lo tanto será útil para estudiantes de ciencias exactas que trabajen con física de plasmas.

Este artículo está dividido en siete secciones. La sección 2 presenta la ecuación MHD básica en función de la velocidad del fluido sin la corriente de desplazamiento. La sección 3 muestra la transformación causada por la corriente de desplazamiento en la ecuación MHD de la velocidad del fluido. En la sección 4 se desarrolla una demostración detallada a partir de la ecuación de la sección 2 hasta llegar a la expresión de la velocidad de fase para cada uno de los tres modos de ondas MHD. Posteriormente, en la Sección 5 se procede de manera similar que en la sección anterior, pero la demostración se realiza a partir de la ecuación de la sección 3 . La Sección [6] discute los resultados encontrados en las secciones 4 y 5 Por último, la sección 7 presenta las conclusiones del trabajo.

\section{Ecuación para la velocidad del fluido despreciando la corriente de desplazamiento}

Las ecuaciones básicas de la MHD son las siguientes [20]:

1. Ecuación de continuidad

$$
\frac{\partial \rho_{m}}{\partial t}+\nabla \cdot\left(\rho_{m} \vec{u}\right)=0
$$


donde $\rho_{m}$ es la densidad del fluido y $\vec{u}$ es la velocidad media del fluido;

2. Ecuación de movimiento derivada de la segunda ley de Newton considerando la fuerza de Lorentz

$$
\rho_{m} \frac{\partial \vec{u}}{\partial t}+\rho_{m}(\vec{u} \cdot \nabla) \vec{u}=-\nabla p+\vec{J} \times \vec{B}
$$

con $\vec{B}$ el campo magnético, $\vec{J}$ la densidad de corriente y $p$ la presión del fluido;

3. Ecuación de conservación de la energía en un proceso termodinámico adiabático $\left(p \rho_{m}^{-\gamma}=\right.$ constante)

$$
\nabla p=v_{s}^{2} \nabla \rho_{m}
$$

en donde $v_{s}$ es la velocidad del sonido adiabática;

4. Ley de Ampère-Maxwell despreciando la corriente de desplazamiento

$$
\nabla \times \vec{B}=\mu_{0} \vec{J}
$$

5. Ley de Faraday

$$
\nabla \times \vec{E}=-\frac{\partial \vec{B}}{\partial t}
$$

en donde $\vec{E}$ es el campo eléctrico;

6. Ley de Ohm generalizada en la forma simplificada, eso significa que fue despreciando el efecto Hall, $\vec{J}$ no depende del tiempo y la presión se reduce a un escalar donde el gradiente es insignificante [10, 21, a pesar de que estos términos se mantienen en algunas de las otras ecuaciones MHD:

$$
\frac{\vec{J}}{\sigma_{0}}=\vec{E}+\vec{u} \times \vec{B}
$$

con $\sigma_{0}$ la conductividad del fluido. En ausencia de un campo magnético externo, 10 se convierte en la ley de $\mathrm{Ohm} \vec{J}=\sigma_{0} \vec{E}$.

Las ecuaciones (5)-10 pueden ser combinadas en una sola ecuación. Los pasos algebraicos para calcular la ecuación se explican detalladamente en el capítulo 15 del libro de [20].

Para obtener la ecuación del fluido es necesario considerar perturbaciones de pequeña amplitud de los valores de equilibrio del campo magnético $\left(\vec{B}(\vec{r}, t)=\vec{B}_{0}+\right.$ $\left.\vec{B}_{1}(\vec{r}, t)\right)$, de la densidad $\left(\rho_{m}(\vec{r}, t)=\rho_{m 0}+\rho_{m 1}(\vec{r}, t)\right)$ y la velocidad del fluido $\left(\vec{u}(\vec{r}, t)=\vec{u}_{1}(\vec{r}, t)\right)$, tomando en cuenta un fluido en reposo $u_{0}=0$, con densidad $\rho_{m 0}$ y campo magnético $\vec{B}_{0}$ uniforme y constante. De esta manera, de la ecuación (5) se obtiene:

$$
\frac{\partial \rho_{m 1}}{\partial t}+\rho_{m 0}\left(\nabla \cdot \vec{u}_{1}\right)=0 .
$$

Combinando (6), (7) y (8) el resultado es:

$$
\rho_{m 0} \frac{\partial \vec{u}_{1}}{\partial t}+v_{S}^{2} \nabla \rho_{m 1}-\frac{\left(\nabla \times \vec{B}_{1}\right) \times \vec{B}_{0}}{\mu_{0}}=0 .
$$

Las ecuaciones (9) y 10 derivan en:

$$
\frac{\partial \vec{B}_{1}}{\partial t}-\nabla \times\left(\vec{u}_{1} \times \vec{B}_{0}\right)=0 .
$$

La ecuación (11) se deriva en función del tiempo para posteriormente sustituir en ella $\sqrt{12}$ y $(13)$. Se considera la solución $\vec{u}_{1}(\vec{r}, t)=\vec{u}_{1} \exp (\iota \vec{k} \cdot \vec{r}-\iota \omega t)$ de una onda plana que permite sustituir $\nabla=\iota \vec{k}$ y $\partial / \partial t=-\iota \omega$, además de la identidad vectorial $\vec{A} \times(\vec{B} \times \vec{C})=$ $(\vec{A} \cdot \vec{C}) \vec{B}-(\vec{A} \cdot \vec{B}) \vec{C}$. El resultado final es la ecuación para la velocidad del fluido que tiene la siguiente expresión:

$$
\begin{gathered}
-\omega^{2} \vec{u}_{1}+\left(v_{s}^{2}+v_{A}^{2}\right)\left(\vec{k} \cdot \vec{u}_{1}\right) \vec{k}+ \\
+\left(\vec{k} \cdot \vec{v}_{A}\right)\left[\left(\vec{k} \cdot \vec{v}_{A}\right) \vec{u}_{1}-\left(\vec{v}_{A} \cdot \vec{u}_{1}\right) \vec{k}-\left(\vec{k} \cdot \vec{u}_{1}\right) \vec{v}_{A}\right]=0 .
\end{gathered}
$$

Vale la pena resaltar que fue despreciada la corriente de desplazamiento cuando se calculó (14). En la siguiente sección se considera el efecto de la corriente de desplazamiento en la ecuación (14).

\section{Ecuación para la velocidad del fluido considerando la corriente de desplazamiento}

Al considerar la corriente de desplazamiento, la ecuación (8) se escribe como

$$
\nabla \times \vec{B}=\mu_{0} \vec{J}+\mu_{0} \epsilon_{0} \frac{\partial \vec{E}}{\partial t} .
$$

Despejando $\vec{J}$ de la ecuación anterior y usando la ecuación 10 para el campo eléctrico $\vec{E}$, se obtiene la siguiente expresión:

$$
\vec{J}=\frac{1}{\mu_{0}}\left(\nabla \times \vec{B}+\mu_{0} \epsilon_{0} \frac{\partial}{\partial t}(\vec{u} \times \vec{B})\right) .
$$

Posteriormente la expresión (16) se sustituye en (6) y de forma similar a la sección anterior, se procede a combinar las ecuaciones (5)-(10). El lector puede encontrar todo el desarrollo algebraico en el capítulo 15 del libro [20]. Al final, se llega a la ecuación para la velocidad del fluido que tiene la siguiente expresión:

$$
\begin{aligned}
& -\omega^{2}\left[\left(1+\frac{v_{A}^{2}}{c^{2}}\right) \vec{u}_{1}-\left(\vec{v}_{A} \cdot \vec{u}_{1}\right) \frac{\vec{v}_{A}}{c^{2}}\right]+\left(v_{s}^{2}+v_{A}^{2}\right)\left(\vec{k} \cdot \vec{u}_{1}\right) \vec{k}+ \\
& +\left(\vec{k} \cdot \vec{v}_{A}\right)\left[\left(\vec{k} \cdot \vec{v}_{A}\right) \vec{u}_{1}-\left(\vec{v}_{A} \cdot \vec{u}_{1}\right) \vec{k}-\left(\vec{k} \cdot \vec{u}_{1}\right) \vec{v}_{A}\right]=0 .
\end{aligned}
$$

Se puede observar que al comparar (17) con (14), la única diferencia radica en el primer término de la izquierda, en el factor que multiplica $\omega^{2}$. Sin embargo, en las próximas secciones mostraremos que este factor afecta las expresiones de la velocidad de fase de las ondas MHD existentes en un plasma. 


\section{Modos de ondas MHD despreciando la corriente de desplazamiento}

En esta sección se lleva a cabo un procedimiento algebraico sobre la ecuación (14), considerando el caso de propagación de ondas magneto-acústicas en un plasma, en donde el vector de onda tiene una dirección arbitraria con respecto al campo magnético.

Antes de iniciar el desarrollo algebraico de (14), es útil definir un sistema de coordenadas cartesianas, de tal forma que el eje $y$ sea perpendicular al plano definido por la dirección $\vec{k}$ y por el vector de inducción magnética $\vec{B}_{0}$, escogiendo $z$ a lo largo de $\vec{B}_{0}$, tal y como se muestra en la Figura 1

Denotando el ángulo entre $\vec{k}$ y $\vec{B}_{0}$ por $\theta$, y los vectores unitarios por $(\hat{i}, \hat{j}, \hat{k})$, tenemos que:

$$
\begin{aligned}
\vec{k} & =k(\sin \theta \hat{i}+\cos \theta \hat{k}), \\
\vec{v}_{A} & =v_{A} \hat{k}, \\
\vec{u}_{1} & =u_{1 x} \hat{i}+u_{1 y} \hat{j}+u_{1 z} \hat{k}, \\
\vec{k} \cdot \vec{v}_{A} & =k v_{A} \cos \theta, \\
\vec{k} \cdot \vec{u}_{1} & =k\left(u_{1 x} \sin \theta+u_{1 z} \cos \theta\right) .
\end{aligned}
$$

Para continuar con el análisis algebraico, es adecuado denotar los miembros de (14) con números romanos $I, I I, I I I, I V, V$ y $V I$ como sigue:

$$
\begin{aligned}
& \underbrace{-\omega^{2} \overrightarrow{u_{1}}}_{I}+\underbrace{\left(v_{s}^{2}+v_{A}^{2}\right)\left(\vec{k} \cdot \overrightarrow{u_{1}}\right) \vec{k}}_{I I}+\underbrace{\left(\vec{k} \cdot \overrightarrow{v_{A}}\right)}_{I I I} \\
& (\underbrace{\left(\vec{k} \cdot \overrightarrow{v_{A}}\right) \overrightarrow{u_{1}}}_{I V}-\underbrace{\left(\overrightarrow{v_{A}} \cdot \overrightarrow{u_{1}}\right) \vec{k}}_{V}-\underbrace{\left(\vec{k} \cdot \overrightarrow{u_{1}}\right) \overrightarrow{v_{A}}}_{V I})=0 .
\end{aligned}
$$

Aunque 23 parece ser muy complicada de resolver, ella conduce a soluciones simples para ondas que se propagan en direcciones paralelas o perpendiculares al campo magnético.

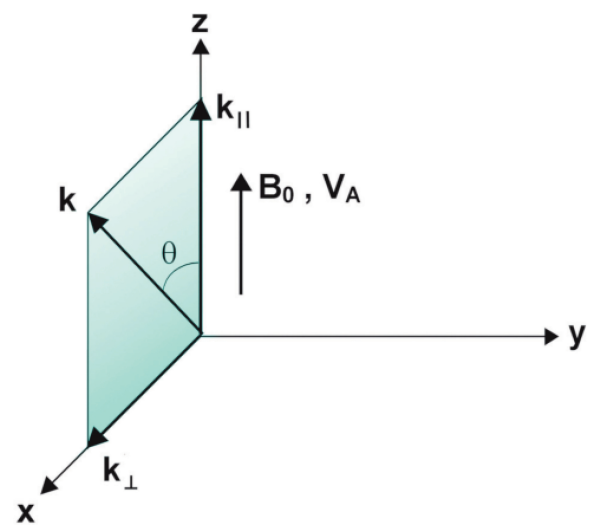

Figura 1: Sistema de coordenadas cartesianas mostrando la orientación relativa de los vectores $\vec{k}$ y $\vec{B}_{0}$. Fuente: Fig. 6 , pag. 386 de [20].
Volviendo a (23), remarcamos que fue separada por partes para una mejor comprensión. Trabajaremos con cada una de las partes por separado sustituyendo las ecuaciones (18)-22) en (23). En la parte $I$,

$$
I=-\omega^{2} u_{1 x} \hat{i}-\omega^{2} u_{1 y} \hat{j}-\omega^{2} u_{1 z} \hat{k} .
$$

En la parte $I I$,

$$
\begin{aligned}
I I= & k^{2}\left(v_{s}^{2}+v_{A}^{2}\right)\left(u_{1 x} \sin \theta+u_{1 z} \cos \theta\right) \\
& (\sin \theta \hat{i}+\cos \theta \hat{k}) \\
= & k^{2}\left(v_{s}^{2}+v_{A}^{2}\right) \sin \theta\left(u_{1 x} \sin \theta+u_{1 z} \cos \theta\right) \hat{i}+0 \hat{j} \\
& +k^{2}\left(v_{s}^{2}+v_{A}^{2}\right) \cos \theta\left(u_{1 x} \sin \theta+u_{1 z} \cos \theta\right) \hat{k} .
\end{aligned}
$$

Luego,

$$
I I I=k v_{A} \cos \theta
$$

y $I V$ se escribe como,

$$
I V=k v_{A} \cos \theta u_{1 x} \hat{i}+k v_{A} \cos \theta u_{1 y} \hat{j}+k v_{A} \cos \theta u_{1 z} \hat{k} .
$$

El miembro $V$ se transforma de la siguiente manera:

$$
\begin{aligned}
V & =-v_{A} u_{1 z} k(\sin \theta \hat{i}+0 \hat{j}+\cos \theta \hat{k}) \\
& =-v_{A} u_{1 z} \sin \theta k \hat{i}+0 \hat{j}-\left(v_{A} u_{1 z} \cos \theta k\right) \hat{k} .
\end{aligned}
$$

Y por último, $V I$ resulta en:

$$
\begin{aligned}
V I & =-k\left(u_{1 x} \sin \theta+u_{1 z} \cos \theta\right) v_{A} \hat{k} \\
& =0 \hat{i}+0 \hat{j}-k v_{A}\left(u_{1 x} \sin \theta+u_{1 z} \cos \theta\right) \hat{k} .
\end{aligned}
$$

Ahora, tenemos que sustituir todos ellos, del $I$ al $V I$, en 23) y hacer los cálculos para cada una de las componentes. Comenzando por el eje $x$ :

$$
\begin{aligned}
& -\omega^{2} u_{1 x}+k^{2}\left(v_{s}^{2}+v_{A}^{2}\right) \sin \theta\left(u_{1 x} \sin \theta+u_{1 z} \cos \theta\right) \\
& +k v_{A} \cos \theta\left[k v_{A} \cos \theta u_{1 x}-k v_{A} \sin \theta u_{1 z}\right]=0 \\
& -\omega^{2} u_{1 x}+k^{2}\left(v_{s}^{2}+v_{A}^{2}\right) \sin ^{2} \theta u_{1 x}+ \\
& +k^{2}\left(v_{s}^{2}+v_{A}^{2}\right) \sin \theta \cos \theta u_{1 z}+ \\
& +k^{2} v_{A}^{2} \cos ^{2} \theta u_{1 x}-k^{2} v_{A}^{2} \sin \theta \cos \theta u_{1 z}=0
\end{aligned}
$$

Continuando el desarrollo matemático,

$$
\begin{aligned}
u_{1 x}[ & \left.-\omega^{2}+k^{2}\left(v_{s}^{2}+v_{A}^{2}\right) \sin ^{2} \theta+k v_{A}^{2} \cos ^{2} \theta\right]+ \\
& +u_{1 z}\left[k^{2}\left(v_{s}^{2}+v_{A}^{2}\right) \sin \theta \cos \theta-k^{2} v_{A}^{2} \sin \theta \cos \theta\right]=0, \\
u_{1 x}[ & -\omega^{2}+k^{2} v_{s}^{2} \sin ^{2} \theta+k^{2} v_{A}^{2} \underbrace{\left(\sin ^{2} \theta+\cos ^{2} \theta\right)}_{=1}] \\
& +u_{1 z}\left[k^{2} v_{s}^{2} \sin \theta \cos \theta+\right. \\
& +\underbrace{k^{2} v_{A}^{2} \sin \theta \cos \theta}-\underline{k}^{2} v_{A}^{2} \sin \theta \cos \theta]=0 .
\end{aligned}
$$


Finalmente, la ecuación para el eje $x$ es la siguiente:

$$
\begin{array}{r}
u_{1 x}\left[-\omega^{2}+k^{2} v_{A}^{2}+k^{2} v_{s}^{2} \sin ^{2} \theta\right] \\
+u_{1 z}\left[k^{2} v_{s}^{2} \sin \theta \cos \theta\right]=0 .
\end{array}
$$

Para el eje $y$ tenemos:

$$
\begin{aligned}
& -\omega^{2} u_{1 y}+0+k v_{A} \cos \theta\left[k v_{A} \cos \theta u_{1 y}-0-0\right]=0 \\
& u_{1 y}\left(-\omega^{2}+k^{2} v_{A}^{2} \cos ^{2} \theta\right)=0 .
\end{aligned}
$$

Para el eje $z$ también hay que hacer desarrollo algebraico:

$$
\begin{aligned}
-\omega^{2} u_{1 z} & +k^{2}\left(v_{s}^{2}+v_{A}^{2}\right) \cos \theta\left(u_{1 x} \sin \theta+u_{1 z} \cos \theta\right) \\
& +k v_{A} \cos \theta\left[k v_{A} \cos \theta u_{1 z}-k v_{A} \cos \theta u_{1 z}\right. \\
& \left.-k v_{A}\left(u_{1 x} \sin \theta+u_{1 z} \cos \theta\right)\right]=0, \\
-\omega^{2} u_{1 z} & +k^{2}\left(v_{s}^{2}+v_{A}^{2}\right) \cos \theta \sin \theta u_{1 x} \\
& +k^{2}\left(v_{s}^{2}+v_{A}^{2}\right) \cos ^{2} \theta u_{1 z}+k^{2} v_{A}^{2} \cos ^{2} \theta u_{1 z} \\
& -k^{2} v_{A}^{2} \cos ^{2} \theta u_{1 z}-k^{2} v_{A}^{2} \cos \theta \sin \theta u_{1 x} \\
& -k^{2} v_{A}^{2} \cos ^{2} \theta u_{1 z}=0 .
\end{aligned}
$$

Continuando el desarrollo matemático,

$$
\begin{aligned}
& u_{1 x}\left[k^{2}\left(v_{s}^{2}+v_{A}^{2}\right) \cos \theta \sin \theta-k^{2} v_{A}^{2} \cos \theta \sin \theta\right] \\
& +u_{1 z}\left[-\omega^{2}+k^{2}\left(v_{s}^{2}+v_{A}^{2}\right) \cos ^{2} \theta+\underline{k^{2} v_{A}^{2} \cos ^{2} \theta}\right. \\
& \left.-k^{2} v_{A}^{2} \cos ^{2} \theta-k^{2} v_{A}^{2} \cos ^{2} \theta\right]=0, \\
& u_{1 x}\left[k^{2} v_{s}^{2} \cos \theta \sin \theta+\underline{k^{2} v_{A}^{2}} \cos \theta \sin \theta\right. \\
& \left.-k^{2} v_{A}^{2} \cos \theta \sin \theta\right]+u_{1 z}\left[-\omega^{2}+k^{2} v_{s}^{2} \cos ^{2} \theta\right. \\
& \left.+k^{2} v_{A}^{2} \cos ^{2} \theta-k^{2} v_{A}^{2} \cos ^{2} \theta\right]=0 .
\end{aligned}
$$

Y llegamos a la ecuación reducida para el eje $z$ :

$$
u_{1 x}\left[k^{2} v_{s}^{2} \sin \theta \cos \theta\right]+u_{1 z}\left[-\omega^{2}+k^{2} v_{s}^{2} \cos ^{2} \theta\right]=0 .
$$

En (31), si $u_{1 y} \neq 0$, tenemos oscilaciones perpendiculares a $\vec{k}$ y $\overrightarrow{B_{0}}$, o sea, una onda linealmente polarizada. De $-\omega^{2}+k^{2} v_{A}^{2} \cos ^{2} \theta=0$, tenemos que:

$$
v_{p h}=\frac{\omega}{k}= \pm v_{A} \cos \theta,
$$

de la ecuación anterior sí $\theta=0$, entonces $\vec{v}_{p h}=\vec{v}_{A} \| \vec{B}_{0}$. Ahora, sí $\theta=90, v_{p h}=0$, y entonces (33) es llamada onda de Alfvén pura.

De las ecuaciones de los ejes $x$ y $z$, lo mas conveniente es escribir 30 y 32 en forma matricial $A u=0$. Donde la matriz $A$ se escribe:

$$
\left(\begin{array}{cc}
-\omega^{2}+k^{2} v_{A}^{2}+k^{2} v_{s}^{2} \sin ^{2} \theta & k^{2} v_{s}^{2} \sin \theta \cos \theta \\
k^{2} v_{s}^{2} \sin \theta \cos \theta & -\omega^{2}+k^{2} v_{s}^{2} \cos ^{2} \theta
\end{array}\right)
$$

y el vector columna $u$ es:

$$
\left(\begin{array}{l}
u_{1 x} \\
u_{1 z}
\end{array}\right)
$$

Para obtener una solución no trivial es necesario que $u_{1 x} \neq 0$ y $u_{1 z} \neq 0$, esto es, que el determinante de $A$ sea igual a cero $(|A|=0)$. Calculando el determinante obtenemos lo siguiente:

$$
\begin{aligned}
\left(-\omega^{2}\right. & \left.+k^{2} v_{A}^{2}+k^{2} v_{s}^{2} \sin ^{2} \theta\right)\left(-\omega^{2}+k^{2} v_{s}^{2} \cos ^{2} \theta\right) \\
& -k^{4} v_{s}^{4} \sin ^{2} \theta \cos ^{2} \theta=0, \\
\omega^{4}-\omega^{2} & k^{2} v_{s}^{2} \cos ^{2} \theta-\omega^{2} k^{2} v_{A}^{2}+k^{4} v_{s}^{2} v_{A}^{2} \cos \theta \\
& -\omega^{2} k^{2} v_{s}^{2} \sin ^{2} \theta+\underline{k^{4} v_{s}^{4} \sin ^{2} \theta \cos ^{2} \theta} \\
& -k^{4} v_{s}^{4} \sin ^{2} \theta \cos ^{2} \theta=0
\end{aligned}
$$

y

$$
\begin{gathered}
\omega^{4}-\omega^{2} k^{2} v_{s}^{2} \underbrace{\left(\cos ^{2} \theta+\sin ^{2} \theta\right)}_{1} \\
-\omega^{2} k^{2} v_{A}^{2}+k^{4} v_{s}^{2} v_{A}^{2} \cos ^{2} \theta=0, \\
\omega^{4}-\left(v_{s}^{2}+v_{A}^{2}\right) \omega^{2} k^{2}+k^{4} v_{s}^{2} v_{A}^{2} \cos ^{2} \theta=0 .
\end{gathered}
$$

Dividiendo ambos lados por $\frac{1}{k^{4}}$, tenemos que:

$$
\left(\frac{\omega}{k}\right)^{4}-\left(v_{s}^{2}+v_{A}^{2}\right)\left(\frac{\omega}{k}\right)^{2}+v_{s}^{2} v_{A}^{2} \cos ^{2} \theta=0 .
$$

Definiendo $X=\left(\frac{\omega}{k}\right)^{2}$ en la ecuación anterior, obtenemos una ecuación de segundo grado para $X$ :

$$
X^{2}-\left(v_{s}^{2}+v_{A}^{2}\right) X+v_{s}^{2} v_{A}^{2} \cos ^{2} \theta=0 .
$$

Resolviendo la ecuación (37) obtenemos las soluciones:

$$
X_{1}=\frac{1}{2}\left(v_{s}^{2}+v_{A}^{2}\right)+\frac{1}{2}\left[\left(v_{s}^{2}+v_{A}^{2}\right)^{2}-4 v_{s}^{2} v_{A}^{2} \cos ^{2} \theta\right]^{\frac{1}{2}}
$$

denotado como modo rápido de la onda MHD y

$$
X_{2}=\frac{1}{2}\left(v_{s}^{2}+v_{A}^{2}\right)-\frac{1}{2}\left[\left(v_{s}^{2}+v_{A}^{2}\right)^{2}-4 v_{s}^{2} v_{A}^{2} \cos ^{2} \theta\right]^{\frac{1}{2}}
$$

es el modo lento.

Por simplicidad es mejor escribir (38) y (39) en una sola ecuación de la velocidad de fase $v_{p h}= \pm \sqrt{X_{1,2}}$ de la onda. Esto es,

$$
v_{p h}= \pm \sqrt{\frac{1}{2}\left(v_{M}^{2} \pm \sqrt{v_{M}^{4}-4 v_{s}^{2} v_{A}^{2} \cos ^{2} \theta}\right)},
$$

donde el signo \pm fuera de la raíz, cambia la dirección de propagación de la onda, mientras que el signo \pm dentro de la raíz indica si es un modo rápido o lento.

El lector también puede encontrar las ecuaciones (33) y (40) en [20] (ecuaciones (5.10) y (5.13) del Cap. 15) y en [5] (Ecuaciones (2.19) y (2.20) del Cap. 2). 


\section{Modos de ondas MHD considerando la corriente de desplazamiento}

La demostración se inicia a partir de (17). Esta ecuación solamente se diferencia de (14) en la expresión del primer término de la izquierda. Esto significa que se pueden utilizar los resultados de la sección anterior para los términos del $I I$ al $V I$ en (23). O sea, la ecuación (17) queda reescrita de la siguiente manera:

$$
\begin{aligned}
& -\omega^{2}\left[\left(1+\frac{v_{A}^{2}}{c^{2}}\right) u_{1}-\left(v_{A} \cdot u_{1}\right) \frac{v_{A}}{c^{2}}\right] \\
& +I I+I I I+I V+V+V I=0 .
\end{aligned}
$$

En (41) se sustituyen (18)- 22 y solamente es necesario trabajar muy poco con el primer término de la ecuación. El resultado es:

$$
-\omega^{2}\left(1+\frac{v_{A}^{2}}{c^{2}}\right) u_{1 x} \widehat{i}-\omega^{2}\left(1+\frac{v_{A}^{2}}{c^{2}}\right) u_{1 y} \widehat{j}-\omega^{2} u_{1 z} \widehat{k} .
$$

Ahora nuevamente vamos separar por ejes, el eje $z$ permanece inalterado y se comienza por el eje $x$ :

$$
\begin{aligned}
u_{1 x} & {\left[-\omega^{2}\left(1+\frac{v_{A}^{2}}{c^{2}}\right)+k^{2} v_{A}^{2}+k^{2} v_{s}^{2} \sin ^{2} \theta\right] } \\
& +u_{1 z}\left[k^{2} v_{s}^{2} \sin \theta \cos \theta\right]=0 .
\end{aligned}
$$

Continuando, el eje $y$ es:

$$
u_{1 y}\left[-\omega^{2}\left(1+\frac{v_{A}^{2}}{c^{2}}\right)+k^{2} v_{A}^{2} \cos \theta\right]=0 .
$$

De (44) si $u_{1 y} \neq 0$, existirán oscilaciones perpendiculares a $\vec{k}$ y $\overrightarrow{B_{0}}$, o sea, la presencia de una onda linealmente polarizada. De esta forma:

$$
-\omega^{2}\left(1+\frac{v_{A}^{2}}{c^{2}}\right)+k^{2} v_{A}^{2} \cos \theta=0,
$$

y se obtiene la expresión para la velocidad de fase de la onda:

$$
v_{p h}=\frac{\omega}{k}= \pm \frac{v_{A} \cos \theta}{\sqrt{1+\frac{v_{A}^{2}}{c^{2}}}} .
$$

En la ecuación anterior se tienen dos casos límite: $i$ ) sí $\theta=0^{\circ}$ entonces $\vec{v}_{p h} \| \vec{B}_{0}$ y tendremos una onda pura de Alfvén; ii) ahora, sí $\theta=90^{\circ}$, entonces $v_{p h}=0$ y por tanto no existe onda.

Para continuar con la demostración, las ecuaciones de los ejes $x$ y $z$ se reescriben convenientemente en forma de matriz:

$$
A_{2,2}\left(\begin{array}{l}
u_{1 x} \\
u_{1 z}
\end{array}\right)=\left(\begin{array}{l}
0 \\
0
\end{array}\right)
$$

donde $A_{2,2}$ es la matriz:

$$
\left(\begin{array}{cc}
-\omega^{2}\left(1+\frac{v_{A}^{2}}{c^{2}}\right) & k^{2} v_{s}^{2} \sin \theta \cos \theta \\
+k^{2} v_{A}^{2}+k^{2} v_{s}^{2} \sin ^{2} \theta & \\
k^{2} v_{s}^{2} \sin \theta \cos \theta & -\omega^{2}+k^{2} v_{s}^{2} \cos ^{2} \theta
\end{array}\right)
$$

Para obtener la solución no-trivial de (47), debe cumplirse que $u_{1 x} \neq 0$ y $u_{1 z} \neq 0$, por tanto es necesario que el determinante de la matriz $A_{2,2}$ se anule. Esto es,

$$
\begin{aligned}
& \left(-\omega^{2}\left(1+\frac{v_{A}^{2}}{c^{2}}\right)+k^{2} v_{A}^{2}+k^{2} v_{s}^{2} \sin ^{2} \theta\right) \\
& \quad\left(-\omega^{2}+k^{2} v_{s}^{2} \cos ^{2} \theta\right) \\
& \quad-\left(k^{2} v_{s}^{2} \sin \theta \cos \theta\right)\left(k^{2} v_{s}^{2} \sin \theta \cos \theta\right)=0 .
\end{aligned}
$$

Se agrupan algunos términos de la siguiente manera:

$$
\begin{aligned}
& \omega^{4}\left(1+\frac{v_{A}^{2}}{c^{2}}\right)-\omega^{2} k^{2} v_{s}^{2} \underbrace{\left(\cos ^{2} \theta+\sin ^{2} \theta\right)}_{=1} \\
& -\frac{\omega^{2} k^{2} v_{s}^{2} v_{A}^{2} \cos ^{2} \theta}{c^{2}}-\omega^{2} k^{2} v_{A}^{2}+k^{4} v_{A}^{2} v_{s}^{2} \cos ^{2} \theta \\
& +k^{4} v_{s}^{4} \sin ^{2} \theta \cos ^{2} \theta-k^{4} v_{s}^{4} \sin ^{2} \theta \cos ^{2} \theta=0 \text {. }
\end{aligned}
$$

Simplificando tenemos que:

$$
\begin{aligned}
& \omega^{4}\left(1+\frac{v_{A}^{2}}{c^{2}}\right)-\left(v_{s}^{2}+v_{A}^{2}+\frac{v_{s}^{2}}{c^{2}} v_{A}^{2} \cos ^{2} \theta\right) \omega^{2} k^{2} \\
& \quad+k^{4} v_{A}^{2} v_{s}^{2} \cos ^{2} \theta=0 .
\end{aligned}
$$

Multiplicando toda la ecuación por $\frac{1}{k^{4}}$ y haciendo el cambio de variable $X=\left(\frac{\omega}{k}\right)^{2}$, tenemos la siguiente ecuación de segundo grado:

$$
\begin{aligned}
(1+ & \left.\frac{v_{A}^{2}}{c^{2}}\right) X^{2}-\left(v_{s}^{2}\left(1+\frac{v_{A}^{2}}{c^{2}} \cos ^{2} \theta\right)+v_{A}^{2}\right) X \\
& +v_{A}^{2} v_{s}^{2} \cos ^{2} \theta=0
\end{aligned}
$$

Continuamos con la resolución de (53), esto es:

$$
\begin{aligned}
\Delta= & \left(v_{s}^{2}\left(1+\frac{v_{A}^{2}}{c^{2}} \cos ^{2} \theta\right)+v_{A}^{2}\right)^{2} \\
& -4\left(1+\frac{v_{A}^{2}}{c^{2}}\right) v_{A}^{2} v_{s}^{2} \cos ^{2} \theta
\end{aligned}
$$

$\mathrm{y}$

$$
X_{1,2}=\frac{\left(v_{s}^{2}\left(1+\frac{v_{A}^{2}}{c^{2}} \cos ^{2} \theta\right)+v_{A}^{2}\right) \pm \sqrt{\Delta}}{2\left(1+\frac{v_{A}^{2}}{c^{2}}\right)} .
$$


Haciendo algunos agrupamientos en la ecuación anterior, obtenemos que:

$$
\begin{aligned}
& X_{1,2}=\frac{1}{2}\left(\frac{\left(1+\frac{v_{A}^{2}}{c^{2}} \cos ^{2} \theta\right)}{1+\frac{v_{A}^{2}}{c^{2}}} v_{s}^{2}+\frac{1}{1+\frac{v_{A}^{2}}{c^{2}}} v_{A}^{2}\right) \\
& \pm \frac{1}{2} \sqrt{\left(\frac{\left(1+\frac{v_{A}^{2}}{c^{2}} \cos ^{2} \theta\right)}{1+\frac{v_{A}^{2}}{c^{2}}} v_{s}^{2}+\frac{1}{1+\frac{v_{A}^{2}}{c^{2}}} v_{A}^{2}\right)-\frac{4 v_{s}^{2} v_{A}^{2} \cos ^{2} \theta}{1+\frac{v_{A}^{2}}{c^{2}}}}
\end{aligned}
$$

Para reducir 56 y escribirla en una forma similar a 40, es conveniente introducir algunos parámetros. La relación entre la velocidad de Alfvén en un sistema de referencia inercial $\left(v_{A}\right)$ y la velocidad de la luz en el vacío (c) se usan para definir $\beta_{A}=v_{A} / c$. Adaptamos el factor de Lorentz para escribirlo en función de la velocidad de Alfvén, denotado por $\gamma_{A}$, donde

$$
\gamma_{A}=\frac{1}{\sqrt{1+\beta_{A}^{2}}} .
$$

Se introduce el parámetro $\gamma_{\theta}$ adimensional:

$$
\gamma_{\theta}^{2}=\gamma_{A}^{2}\left(1+\left(\beta_{A} \cos \theta\right)^{2}\right)
$$

el cual representa ondas magneto-acústicas, además se introduce también una expresión similar a (4) que tiene unidades de velocidad:

$$
v_{M \gamma}^{2}=\gamma_{\theta}^{2} v_{s}^{2}+\gamma_{A}^{2} v_{A}^{2},
$$

esta expresión nos permite definir $v_{M \gamma}$ como la velocidad magneto-acústica anisotrópica-relativista, porque depende de $\theta$ y del valor de $v_{A}$ en relación a $c$. Se puede verificar que para $\theta=90^{\circ}$ y $v_{A} \ll c$ se obtiene (4). El paso final es sustituir (57) y $(59)$ en $(56)$ :

$$
v_{p h}= \pm \sqrt{\frac{1}{2}\left(v_{M \gamma}^{2} \pm \sqrt{v_{M \gamma}^{4}-4 \gamma_{A}^{2} v_{s}^{2} v_{A}^{2} \cos ^{2} \theta}\right)},
$$

donde el signo al frente de la raíz cuadrada externa, cambia la dirección de propagación de la onda. Comparando con 40, la ecuación anterior representa los modos MHD rápido para el signo positivo en la raíz cuadrada interna y lento cuando el signo es negativo. Además, es importante verificar que para $v_{A}<<c$ las ecuaciones 46 y 60 se transforman en 33 y 40 , respectivamente.

El lector también puede encontrar las ecuaciones 46 y (60) en [5] (Ecuaciones (8.71) y (8.72) del Cap. 8). Sin embargo, podrá verificar que en la referencia anterior no se muestran los pasos algebraicos para obtener 46) y 60. Simplemente son introducidas, para posterior usarlas en el estudio de ondas de Alfvén y ondas magnetoacústicas en las condiciones extremas de temperatura y campo magnético encontradas en el plasma alrededor de un púlsar. Además, resulta conveniente mencionar que las ecuaciones (46) y 60, considerando todas las direcciones de propagación, no aparecen de esta manera en 20, en donde el autor solamente presenta las ecuaciones (ver Ecuaciones (6.11) y (6.13) del Cap. 15) para la propagación de una onda paralela $\left(\operatorname{con} \theta=0^{\circ}\right)$ y perpendicular $\left(\operatorname{con} \theta=90^{\circ}\right)$ al campo magnético.

\section{Resultado y discusión}

Esta sección se inicia con una discusión física sobre la conveniencia de incluir o despreciar la corriente de desplazamiento al estudiar ondas de Alfvén y ondas magneto-acústicas en un determinado sistema físico inmerso en un plasma.

En primer lugar, debemos entender el por qué de la corrección de Maxwell a la ley de Ampère. Esta ley explica la existencia de un campo magnético alrededor de un medio conductor como consecuencia del flujo de carga eléctrica a través del mismo. Sin embargo, en un circuito eléctrico, al incluir un condensador eléctrico o capacitor en la trayectoria de la corriente eléctrica, el flujo de carga entre las placas es interrumpido, pero continua existiendo un campo magnético rodeando la región entre las placas. Maxwell resolvió elegantemente este problema agregando el término $J_{d}$ a la ley de Ampère. O sea, la variación temporal de campo eléctrico en el interior del capacitor, causado por las cargas de polaridades opuestas acumuladas en las placas metálicas, es el responsable de la existencia del campo magnético. Tal resultado, también fue útil para obtener las ecuaciones de ondas electromagnéticas propagándose en el vacío.

En segundo lugar, es evidente que, el término incorporado por Maxwell es una generalización, no una especificación, por tanto, su uso no invalidará el resultado de un determinado modelo. De hecho, las ecuaciones de Maxwell describen por completo los fenómenos electromagnéticos. El problema surge cuando, por conveniencia, generalmente para reducir la complejidad matemática de los modelos (como es explicado en [6]), deseamos ignorar $J_{d}$. En la formulación presentada en este manuscrito, las ecuaciones (46) y 60 pueden usarse en cualquier plasma que cumpla con las condiciones (simplificaciones) adoptadas en la ecuación de Ohm (10). Dejamos claro que al incorporar otros términos en la ley de Ohm, no significa que invalide el uso de $J_{d}$ sino que tendremos aumento en la complejidad del sistema, que lleva hacer un estudio numérico del problema, así como también la inclusión de otros fenómenos físicos tales como inestabilidades y algunos tipos de ondas [18].

Para despreciar $J_{d}$ en los cálculos de un fluido magneto-conductor se debe considerar el orden de magnitud de la velocidad de Alfvén (1) y los valores extremos que puede adoptar. Sí $v_{A}<<c$ en todo el volumen espacio-temporal entonces se puede despreciar $J_{d}$. Como $v_{A}$ es directamente proporcional al valor del campo magnético e inversamente proporcional a la densidad, basta tener conocimiento previo de los valores de estas 
magnitudes, en un determinado plasma, para poder tomar en cuenta o despreciar el valor de $J_{d}$. Por ejemplo, en [6] el autor considera la atmósfera solar en un estado termodinámico isotérmico que le permite adoptar una función exponencial de la densidad en función de la altura. También considera un modelo analítico para reproducir el campo magnético en al región de interés. En ese modelo, como la densidad disminuye exponencialmente, se pueden obtener erróneamente velocidades de Alfvén mayores que c, y como resultado de despreciar $J_{d}$ surge una desestimación del flujo de energía que fluye por la frontera superior. Como las ondas de Alfvén no se propagan en el vacío, ellas serán reflejadas en la frontera superior de regreso para dentro del sistema, lo que no es deseado. Sin embargo, al considerar $J_{d}$, las fronteras son definidas de tal forma que mas allá de la frontera superior, en el vacío, la onda de Alfvén se convertirá en una onda electromagnética y no tendrá necesidad de ser reflejada de regreso al sistema. Otro ejemplo, lo podemos encontrar en plasmas relativistas de electrones e positrones, como en los púlsares y en núcleos activos de galaxias, constituidos por intensos campos magnéticos donde se debe tomar en cuenta $J_{d}$ para estudiar la velocidad de fase de las ondas [5].

Esta sección continua mostrando en los dos paneles superiores de la Fig. 2 la variación de la velocidad de fase en los modos de ondas MHD rápido (color azul), lento (color rojo) y puro (color negro) como función del ángulo $\theta$. El panel (a) corresponde al caso $v_{A}>v_{s} \mathrm{y}$ el (b) a $v_{A}<v_{s}$. Las gráficas fueron diseñadas a partir de (46) y (60), considerando que $v_{A} \ll c$ y variando $\theta$ entre 0 y $90^{\circ}$. Debido a la condición $v_{A} \ll c$, el resultado sería el mismo si usamos (33) y (40). Este resultado coincide con el mostrado en la Fig. 7 pág. 389 de [20]. Ambos paneles ayudan a comprobar que las deducciones presentadas para obtener (33) y (40), a partir de considerar la corriente de desplazamiento $J_{d}$ son correctas. La comprobación de los resultados es una etapa importante en la construcción del conocimiento científico, y resulta una herramienta útil para los estudiantes jóvenes en el área de física.

Analizando con más detalles el panel (a), podemos ver que sí $\theta=0^{\circ}$, el modo rápido y puro presentan la misma velocidad de fase que es igual a la velocidad de Alfvén. Es decir, es una onda de Alfvén desplazándose en la dirección del campo magnético y oscilando en una dirección transversal. La velocidad de fase del modo rápido aumenta conjuntamente con el valor de $\theta$, hasta que en $90^{\circ}$ tendrá el valor $v_{p h}=v_{M \gamma}$ dado por (59), y que se transforma en (4) cuando $v_{A} \ll c$. Los otros dos modos disminuyen hasta el valor de $v_{p h}=0$ en $90^{\circ}$. En el panel (b), comparando con (a), vemos que el modo (a)

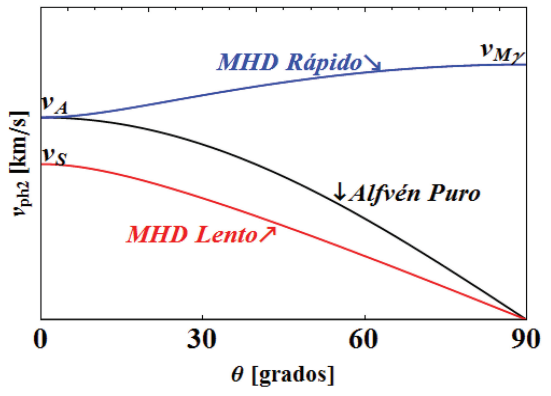

(c)

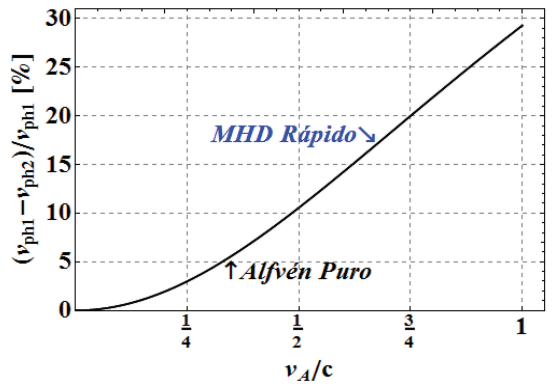

(b)

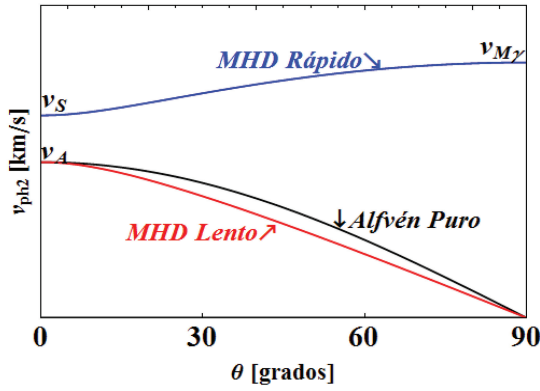

(d)

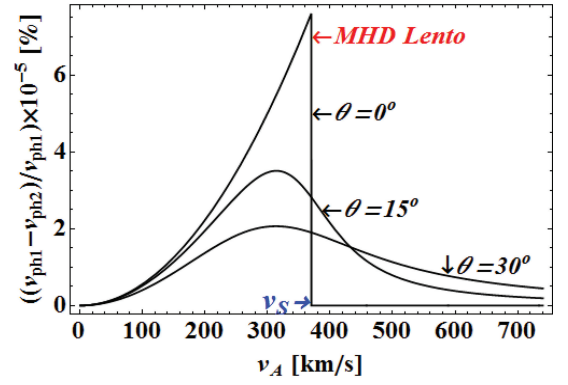

Figura 2: En los paneles superiores se muestran las variaciones de la velocidad de fase en cada uno de los modos de ondas MHD rápido (color azul), lento (color rojo) y puro (color negro) en relación al ángulo $\theta$ entre $\vec{k}$ e $\vec{B}_{0}$, para los casos (a) $v_{A}>v_{s} \mathrm{e}$ (b) $v_{A}<v_{s}$. Los gráficos se realizaron a partir de las ecuaciones (46) y (60), pero el resultado sería el mismo que el mostrado en la Fig. 7, pag 389 de [20], construido con las ecuaciones (33) y (40). Lo anterior se debe a que fue utilizada la condición $v_{A} \ll c$. Los paneles inferiores muestran el porcentaje de variación de la velocidad de fase en cada uno de los tres modos de ondas cuando se considera el efecto de la corriente de desplazamiento, influenciados por la variación de la velocidad de Alfvén cuando $v_{A}>v_{S}$. El panel (c) muestra que los modos rápido y puro están sobrepuestos, la forma del gráfico no depende del valor de $\theta$. El panel (d) muestra el modo de onda lento para tres valores distintos del ángulo $\theta$. 
puro no tiene ninguna alteración. Los modos rápido y lento solamente se alteran cuando $\theta=0^{\circ}$, en este caso para el modo rápido $v_{p h}=v_{s} \mathrm{y}$ para el lento $v_{p h}=v_{A}$.

En los dos paneles inferiores de la Fig. 2 el objetivo es mostrar cómo es la variación de (46) y (60) en relación a (33) y 40 cuando la velocidad de Alfvén aumenta. Esto sería una manera de cuantificar lo importante que es considerar el efecto de la corriente de desplazamiento al momento de calcular la velocidad de fase de cada uno de los modos de ondas MHD. Para realizar esta comparación, usamos la siguiente relación:

$$
P(\%)=\frac{v_{p h 1}-v_{p h 2}}{v_{p h 1}} \times 100 \%
$$

donde el subíndice 1 se refiere a una de las ecuaciones (33) o 40, mientras que el subíndice 2 se refiere a 46 ó 60 .

La ecuación (61) siempre tendrá valores positivos porque el efecto de la corriente de desplazamiento hace que se tenga un límite en el valor máximo de la velocidad de fase, por eso $v_{p h 1}>v_{p h 2}$. Por ejemplo, considerando (46) con $\theta=0^{\circ}$, en el caso hipotético que matemáticamente $v_{A} \rightarrow \infty$ la velocidad de fase será $v_{p h}=c$. Sin embargo, bajo las mismas condiciones en 33 obtenemos que $v_{p h} \rightarrow \infty$. Una forma más clara de ver esto, es cuando suponemos que $v_{A} \approx c$, en las mismas condiciones, tendríamos que 46 deriva en $v_{p h} \approx c / \sqrt{2}$ y 33 da como resultado $v_{p h} \approx c$.

Para usar como ejemplo de comparación, consideremos algunos valores característicos del viento solar en el medio interplanetario, cercanos a la órbita terrestre, esos valores son $B=5 \mathrm{nT}$ y $\rho=5$ protón $/ \mathrm{cm}^{3}$. Para obtener $v_{A} \approx c$ con ese valor de densidad, el campo magnético tendría que ser igual a $B \approx 30733 \mathrm{nT}$. Ya con $B=5$ $\mathrm{nT}$ el valor de la densidad es $0,13 \times$ protón $/ \mathrm{m}^{3}$. Ambos valores son muy poco probables de ser encontrados en la naturaleza. Otro ejemplo sería la fotosfera solar a una altura de $490 \mathrm{~km}$, en donde se pueden observar campo magnéticos del orden de $1 \mathrm{~T}$. Para tales condiciones anteriores las ondas de Alfvén desaparecen [22, 23].

A modo de ejemplo didáctico, en la Fig. 2 mostramos el porcentaje de variación de (61) para los modos de onda MHD puro y rápidos en función de la variación en la relación $v_{A} / c$. Tomando en cuenta el hecho de que consideramos el caso $v_{A}>v_{S}$, no tiene sentido realizar esta discusión para $v_{A}<v_{S}$, porque siempre estaremos en la condición $v_{A} \ll c$ donde el efecto de la corriente de desplazamiento en el valor de la velocidad de fase de la onda es muy pequeño. El valor de $v_{A} / c$ fue extendido intencionalmente hasta el valor máximo 1. Como resultado, se muestra que los modos puro y rápido están superpuestos porque mostraron el mismo comportamiento funcional cuando variamos $v_{A} / c$. Además, se comprobó que la forma de la gráfica no depende del ángulo $\theta$. Observamos que en el intervalo $0<v_{A} / c<1 / 2$ existe un porcentaje de variación de $10 \%$. En $v_{A} / c \approx 1$ este porcentaje es de aproximadamente $29 \%$.
En la Fig. 2d dibujamos 61 para observar el comportamiento del modo MHD lento, este modo a diferencia de los anteriores, depende del ángulo $\theta$. Cuando el ángulo aumenta de $0^{\circ}$ hasta $90^{\circ}$ el porcentaje de variación disminuye. Aún así, este modo prácticamente no se ve influenciado por el efecto de la corriente de desplazamiento, debido a que el porcentaje de variación es del orden de $10^{-5} \%$. El valor máximo de 61 observado en el panel $2 \mathrm{~d}$ es $\approx 8 \times 10^{-5} \%$ cuando $v_{A}=v_{s}$ y $\theta=0^{\circ}$. Para ángulos mayores, el máximo continua estando en $v_{A}=v_{s}$ pero con un valor menor que en el caso $\theta=0^{\circ}$.

\section{Conclusiones}

En la elaboración de este artículo se abordó el tema de los modos de ondas que se obtienen a partir de las ecuaciones básicas de la MHD. Nuestro objetivo fue explicar la importancia que tiene considerar la corriente de desplazamiento en la ley de Ampère-Maxwell, cuando se calcula una ecuación para la velocidad de un fluido. A pesar que este es un tema conocido en física de plasmas, el enfoque que se le dio en este trabajo fue pedagógico durante la presentación del contenido.

Se tomó especial cuidado en mostrar los pasos algebraicos necesarios para desarrollar las ecuaciones, de forma tal que este artículo pueda ser utilizado como material de apoyo en cursos de física de plasmas. Verificamos que en la literatura, solamente se presentaba el desarrollo matemático para llegar hasta la velocidad de fase de los modos de ondas MHD, cuando no se consideraba la corriente de desplazamiento, faltando la presentación de los detalles al considerar esta corriente. Con el objetivo de ayudar a estudiantes de física, mostramos con detalle el desarrollo algebraico para obtener los modos de ondas considerando la corriente de desplazamiento.

La mayor parte de la discusión del artículo se centró alrededor de la interpretación física que resulta de la inclusión de la corriente de desplazamiento y de las diferencias numéricas entre las velocidades de fase de cada modo de onda MHD, al considerar o no la corriente de desplazamiento y en el caso cuando la velocidad de Alfvén aumenta hasta valores cercanos a la velocidad de la luz. La velocidad de Alfvén puede aumentar por tres motivos: el aumento del campo magnético, la disminución de la densidad o por ambos efectos juntos. Para obtener valores próximos a $c$ se necesitan de valores extremos de campo y densidad que en la práctica son difíciles de medir para relacionarlos con la presencia de ondas de Alfvén. Generalmente, esos valores son estimados a través de funciones analíticas que alimentan modelos que permiten estudiar las características de las ondas de Alfvén. Por eso, resultaría útil considerar la corriente de desplazamiento en plasmas relativistas de positrones y electrones de objetos astrofísicos, así como en modelos analíticos que estudian la atmósfera solar. En plasmas fríos no relativistas, como el del medio interplanetario o la magnetosfera de la Tierra, al 
despreciar $J_{d}$, los resultados en la velocidad de fase de la onda siguen siendo físicamente válidos.

Como resultado principal del artículo, mostramos que el efecto de la corriente de desplazamiento es muy importante cuando trabajamos con los modos de ondas MHD puro y rápido en los casos que $v_{A}>v_{s}$. Para el modo de onda MHD lento, la corriente de desplazamiento prácticamente no influye en el valor de la velocidad de fase de la onda cuando la velocidad de Alfvén aumenta. Al considerar la corriente de desplazamiento, sugerimos calcular la velocidad magnetoacústica con la expresión (59) que definimos como la velocidad magnetoacústica anisotrópica-relativista.

\section{Agradecimientos}

A. Ojeda-González desea agradecer al CNPq por su apoyo financiero (número de proyecto 431396/2018-3). J. J. González-Avilés agradece a Cátedras CONACYT, CONACYT LN 314845 y CONACYT-AEM 2017-01292684 por el apoyo parcial a este trabajo. El Servicio de Clima Espacial de México (SCIESMEX) está patrocinado por el Programa Cátedras-CONACYT, proyecto 1045. V. De la Luz agradece a CONACYT por su apoyo financiero (Proyecto de Ciencia Básica con número 254497) y UNAM/PAPIIT TA101920. L. Nunes dos Santos agradece a PROSUC-CAPES por la beca de doctorado en el curso de Física y Astronomía en la UNIVAP. Proyecto vinculado al Grupo de investigación de CNPq "Matemática Aplicada à Fúsica Espacial"

\section{Referências}

[1] R. Fitzpatrick, Maxwell's Equations and the Principles of Electromagnetism, (Infinity Science Press, Hingham, 2008).

[2] W.B. Thompson, em: An Introduction to Plasma Physics, editado por W.B. Thompson (Pergamon, Oxford, 1962).

[3] H. Alfvén, Nature 150, 405 (1942).

[4] H. Alfvén, Rev. Geophys 15, 271 (1977).

[5] N.F. Cramer, Plasma Phys. Control. Fusion.44, 293 (2001).

[6] B. Leroy, Astron. Astrophys.125, 371 (1983).

[7] C.E. Parnell, em: Anais do Lecture Notes in Physics editado por J.P. Rozelot, L. Klein e J.C. Vial (Springer Verlag, Berlin, 2000)

[8] F.J.R. Simões Jr, E. Costa Jr, M.V. Alves e F.R. Cardoso, Rev. Bras. Ensino Fis. 33, 1 (2011).

[9] M. Cristaldo-Oliveira, A. Ojeda-González, L.N. Santos, A. Prestes e A.N. Laurindo-Sousa, Rev. Bras. Ensino Fis.42, e20200297 (2020).

[10] V.M. Souza, M.V.D. Silveira, D. Koga e P.R. Jauer, Rev. Bras. Ensino Fis. 38, e2301 (2016).

[11] K. Schindler, Physics of Space Plasma Activity (Cambridge University Press, Cambridge, 2006).
[12] P.P. Ferreira, S.M.V. Dias, F.R. Cardoso, D. Koga, V.M. Souza, L.E.V. Antunes, G. Farinas, B.M. CuadrosMelgar, W.D. Gonzalez e E.F. dos Santos, Rev. Bras. Ensino Fis. 39, e2307-1 (2017).

[13] H. Alfvén, Ark. Mat. Astr. Fys. 29, 1 (1943).

[14] E. Costa Jr., F.R. Cardoso, F.J.R. Simoes Jr. e M.V. Alves, Rev. Bras. Ensino Fis. 331, 4301 (2011).

[15] A. Ojeda-González, O. Mendes, V.E. Menconi e M.O. Domingues, Geofís. Int. 53, 101 (2014).

[16] A. Prestes, V. Klausner e A. Ojeda-González, Ann. Geophys. 35, 1231 (2017).

[17] J.J. González-Avilés, F.S. Guzmán, V. Fedun, V.G. Verth, R. Sharma, S. Shelyag e S. Regnier, MNRAS 484, 1871 (2019).

[18] P.R. Jauer, C. Wang, V.M. Souza, M.V. Alves, L.R. Alves, M.B. Pádua, J.P. Marchezi, L.A. Da Silva, Z. Liu, H. Li et al., Astrophys. J. 886, 59 (2019).

[19] J.W. Belcher, L. Davis e E.J. Smith, J. Geophys. Res. 74, 2302 (1969).

[20] J.A. Bittencourt, Fundamentals of plasma physics (Pergamon Press, New York, 1986), v. 1

[21] J.A. Bittencourt, Fundamentals of plasma physics (Pergamon Press, New York, 1986), v. 1.

[22] J. Vranjes e M. Kono, Phys. Plasmas 21, 012110 (2014).

[23] J. Vranjes, MNRAS 445, 1614 (2014). 